\title{
El uso potencial de los pinenos como biocombustibles
}

\author{
M.C. Sarai Alejandro Hernández \\ sarai.alejandro@ujat.mx \\ Dr. David Guerrero Zárate \\ david.guerrero@ujat.mx
}

División Académica Multidisciplinaria de Jalpa de Méndez

Universidad Juárez Autónoma de Tabasco

Villahermosa, Tabasco, México

\section{RESUMEN}

Los pinenos son moléculas obtenidas de la trementina, un producto derivado de los pinos.

En la literatura se encuentran trabajos en los que se propone su uso como biocombustible; por lo anterior, en este trabajo se llevó a cabo una comparación entre las propiedades físicoquímicas del $\beta$-pineno con las de alcanos lineales usados como moléculas modelo de los combustibles derivados del petróleo, que son el n-heptano, el n-decano, el ndodecano y el n-hexadecano; los resultados demuestran que las propiedades del $\beta$-pineno son cercanas a las de las moléculas modelo estudiadas y al comparar los valores con los límites establecidos en la normatividad vigente para México, se encontró que es posible utilizar a este monoterpeno bicíclico como un aditivo para combustibles derivados del petróleo. Al usar derivados de la trementina como biocombustibles, se puede tener un proceso sustentable, ya que para la producción de la resina no es necesario talar los pinos, promoviendo así la reforestación de los bosques de coníferas, con la posibilidad de obtener un producto de valor agregado para los propietarios de esas tierras.

Palabras clave: trementina; sustentabilidad; gasolina; diésel; turbosina. 


\title{
The potential use of pinenes as biofuels
}

\begin{abstract}
The pinenes are molecules from turpentine, a product derived from pines. In the literature, it is possible to find papers proposing the use of pinenes as biofuels; by those mentioned above, in this work, we compared the physicochemical properties of $\beta$-pinene with the properties of linear alkanes common used as model molecules for fossil fuels like nheptane, n-decane, n-dodecane, and n-hexadecane. The results show that the properties of $\beta$-pinene are closer to the model molecules studied. When we compare the values obtained with lower and upper limits established in the current regulations for Mexico, we found that it is possible to use this bicyclic monoterpene as an additive for fossil fuels. If we use turpentine-derived substances as biofuels, we could have a sustainable process because for the pine-resin production is not necessary to cut down the conifers, promoting reforestation of forests with the possibility of obtaining a value-added product for the landowners.
\end{abstract}

Keywords: turpentine; sustainability; gasoline; diesel; jet-fuel

Artículo recibido: 02 noviembre. 2021 Aceptado para publicación: 28 noviembre 2021

Correspondencia: david.guerrero@ujat.mx Conflictos de Interés: Ninguna que declarar 


\section{INTRODUCCIÓN}

La resina de pino es considerada como el oro líquido de los bosques de coníferas (Girón, 2021), ya que al someterla a un proceso de destilación por arrastre de vapor es posible obtener un sólido denominado colofonia y un destilado, llamado trementina (Mata, Villanueva, \& Henríquez, 2018). Desde tiempos antiguos se conoce el uso de la trementina en como solvente y más recientemente, como combustible para motocicletas, cuando Soichiro Honda comenzó con la fabricación de sus motores de 50 cc, en 1946 (Martins \& Brito, 2020). La trementina está compuesta principalmente por dos isómeros monoterpénicos, el $\alpha$-pineno y el $\beta$-pineno (Mirov, Zavarin, \& Snajberk, 1966). Estos terpenos son hidrocarburos bicíclicos con diez carbonos $\left(\mathrm{C}_{10} \mathrm{H}_{16}\right)$, diferenciados por su estructura molecular y su interacción con la luz polarizada, véase la Figura 1.

El proceso de obtención de la trementina comienza con la resinación de los pinos, que consiste en hacer incisiones en la corteza de los árboles para recolectar la resina (Toledo \& Chávez, 2006). Posteriormente, la resina se somete a un proceso de destilación por arrastre de vapor, en donde el vapor de agua procedente de la caldera transfiere energía a los componentes volátiles de la resina, transportándose en forma de vapor hacia la sección de condensación, donde se usa agua de enfriamiento para obtener una mezcla líquida orgánico-acuosa, que finalmente se separa por decantación, obteniéndose por la sección del fondo agua con trazas de los componentes orgánicos y en el sobrenadante la trementina. En la Figura 2, se muestra un esquema simplificado del proceso.

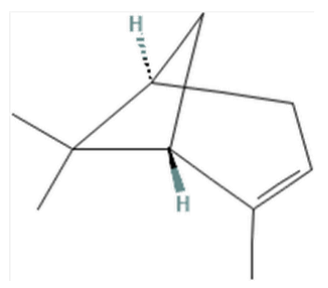

$(+)-\alpha-$ pineno

C.A.S.: $7785-70-8$

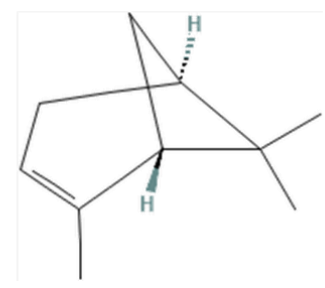

$(-)-\alpha-$ pineno C.A.S.: $7785-26-4$

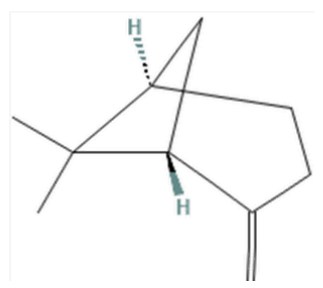

$(+)-\beta$-pineno

C.A.S.: $19902-08-0$

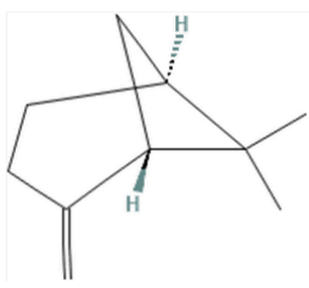

$(-)-\beta$-pineno

C.A.S.: $18172-67-3$

Figura 1. Estructuras moleculares y número de registro del Chemical Abstract Service

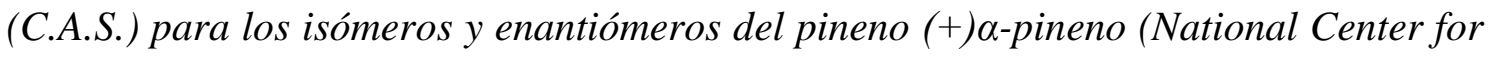
Biotechnology Information, 2021d), (-)a-pineno (National Center for Biotechnology Information, 2021c), (+)ß-pineno (National Center for Biotechnology Information, 2021a), (-)ß-pineno(National Center for Biotechnology Information, 2021b). 


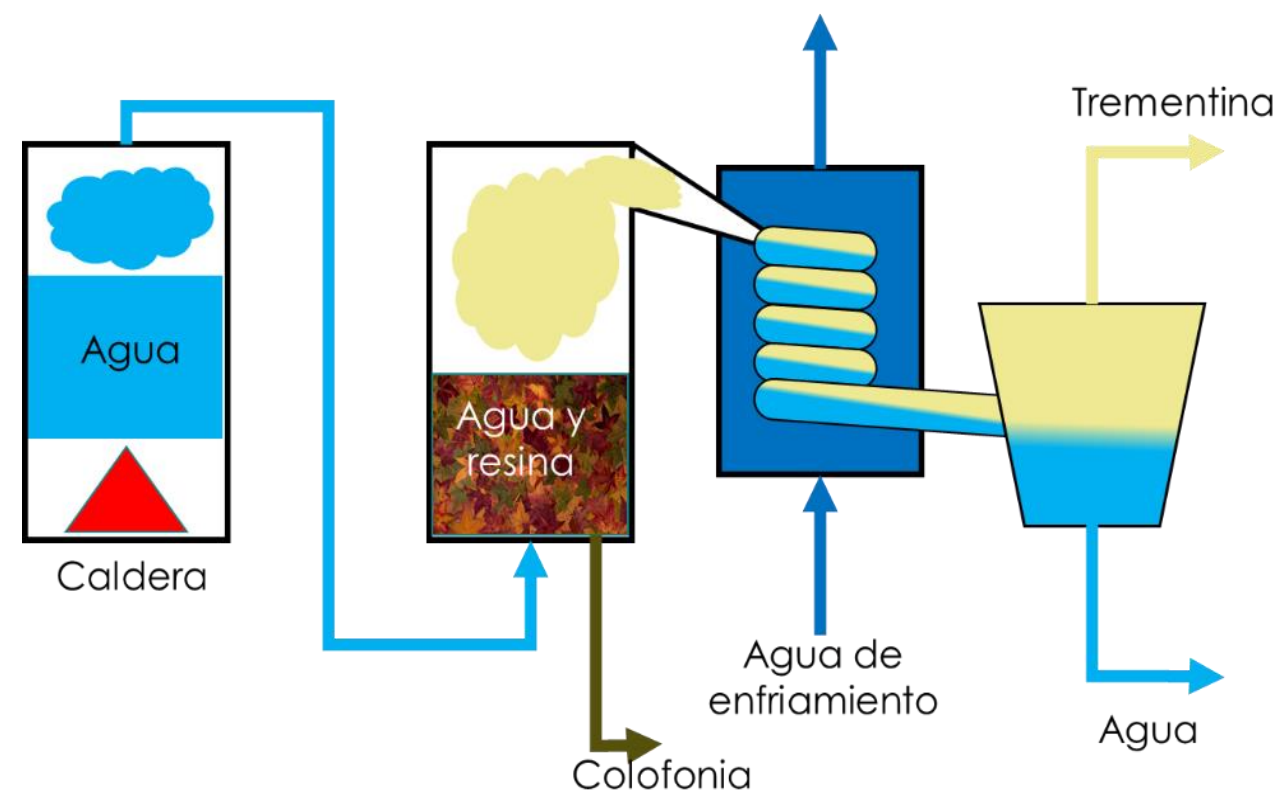

Figura 2. Proceso de producción de la trementina a partir de la resina de pinos, mediante destilación por arrastre de vapor.

El uso de los pinenos como bio-combustibles ha sido estudiado recientemente desde diversas perspectivas, por ejemplo, el uso de la trementina en motores diésel (Anand, Saravanan, \& Srinivasan, 2010) o como bio-componente en la gasolina (Knuuttila, 2013), la síntesis de bio-queroseno a partir de los terpenos presentes en la trementina (Hudaya, Widjaja, Rionardi, \& Soerawidjaja, 2016), la dimerización del $\beta$-pineno para obtener una molécula de alta densidad energética con potencial uso como turbosina (Jung et al., 2016) y el uso de trementina hidrogenada u oxigenada para automóviles (Donoso, García, Ballesteros, Lapuerta, \& Canoira, 2021).

Cuando se desea estudiar desde un punto de vista básico el comportamiento molecular de las gasolinas, el diésel y la turbosina, se recurre al uso de moléculas modelo de referencia. En la Tabla 1, se muestran las moléculas representativas para diversos combustibles (Pitz et al., 2007). 
Tabla 1. Moléculas modelo empleadas para estudiar el comportamiento molecular de diversos petrolíferos

\begin{tabular}{llc}
\hline Petrolífero & \multicolumn{1}{c}{ Molécula modelo } & $\begin{array}{c}\text { Número de } \\
\text { registro C.A.S. }\end{array}$ \\
\hline \multirow{2}{*}{ Gasolina } & $n$-Heptano & $142-82-5$ \\
Diésel & 2,2,4-Trimetilpentano & $540-84-1$ \\
& $n$-Hexadecano & $544-76-3$ \\
& $2,2,4,4,6,8,8-H e p t a m e t i l n o n a n o$ & $4390-04-9$ \\
& $n$-Decano & $124-18-5$ \\
& $n$-Dodecano & $112-40-3$ \\
Turbosina (Honnet, Seshadri, & $n$-Tetradecano & $629-59-4$ \\
Niemann, \& Peters, 2009) & 2,2,4,4,6,8,8-Heptametilnonano & $4390-04-9$ \\
& Metilciclohexano & $108-87-2$ \\
& Butilcicloho & $95-47-6$ \\
& Butilbenceno & $1687-93-9$ \\
& Trimetilbenceno & $104-51-8$ \\
& Tetralín & $25551-13-7$ \\
& 1-Metilnaftaleno & $119-64-2$ \\
\hline
\end{tabular}

En este trabajo se realizó un estudio comparativo entre las propiedades fisicoquímicas y de transporte del $\beta$-pineno con respecto a los hidrocarburos parafínicos usados como moléculas modelo de los petrolíferos: $n$-heptano $\left(n-\mathrm{C}_{7}\right), n$-decano $\left(n-\mathrm{C}_{10}\right), n$-dodecano $(n$ $\mathrm{C}_{12}$ ) y $n$-hexadecano $\left(n-\mathrm{C}_{16}\right)$, con la finalidad de conocer el uso potencial de este monoterpeno bicíclico como un biocombustible.

\section{ESTRATEGIAS METODOLÓGICAS}

Las densidades y viscosidades cinemáticas a presión atmosférica para el $n-\mathrm{C}_{7}, n-\mathrm{C}_{10}$ y $n$ $\mathrm{C}_{12}$ se obtuvieron de la base de datos Propiedades Termofísicas de Sistemas Fluidos del National Institute of Standards and Technology (NIST) (Lemmon, McLinden, \& Friend, 2021), en el intervalo de 293.15 a $363.15 \mathrm{~K}$, con incrementos de $5 \mathrm{~K}$; en el caso del $n$ $\mathrm{C}_{16}$, se utilizaron los datos reportados por (Luning Prak, Cowart, McDaniel, \& Trulove, 2016). El poder calorífico para los hidrocarburos parafínicos se obtuvo del Libro del Web de Química del NIST (Burgess, 2021).

Para el $\beta$-pineno, la densidad y viscosidad cinemática a presión atmosférica se midieron en un densímetro digital de tubo oscilante modelo DMA 4100 M y en viscosímetro digital Stabinger modelo SVM 2001, ambos de la marca Anton Paar; el intervalo de temperatura para ambas propiedades fue de 293.15 a $363.15 \mathrm{~K}$, con incrementos de $5 \mathrm{~K}$. El poder 
calorífico se determinó usando una bomba calorimétrica de oxígeno modelo A4000.

En México, la legislación que establece las características de calidad para los petrolíferos es la Norma Oficial Mexicana NOM-016-CRE-2016 (Comisión Reguladora de Energía, 2016), que es aplicable a las gasolinas, turbosinas, diésel automotriz, entre otros. Por lo anterior, además de hacer la comparación entre moléculas modelo y el $\beta$-pineno, se llevará a cabo un cotejo con los valores establecidos en dicha norma para las propiedades de los petrolíferos utilizadas en este trabajo.

\section{RESULTADOS Y DISCUSIÓN}

En la Figura 3, se puede apreciar la dependencia de las densidades para cuatro hidrocarburos parafínicos y el $\beta$-pineno, con respecto a la temperatura. Se puede observar que la sustancia más densa es el $\beta$-pineno, mientras que los alcanos lineales incrementan su densidad al aumentar el número de carbonos en su cadena. Las líneas discontinuas corresponden a los valores mínimo y máximo para la densidad de la turbosina, establecido en la NOM-016-CRE-2016; se puede observar que el $\beta$-pineno no podría usarse directamente como un sustituto de la turbosina, puesto que, al ser más denso, provocaría una mayor carga en los depósitos de combustible de los aviones, sin embargo, tiene un uso potencial como sustituto de la gasolina y el diésel, ya que para dichos petrolíferos esta propiedad sólo debe ser reportada, de acuerdo con la norma.

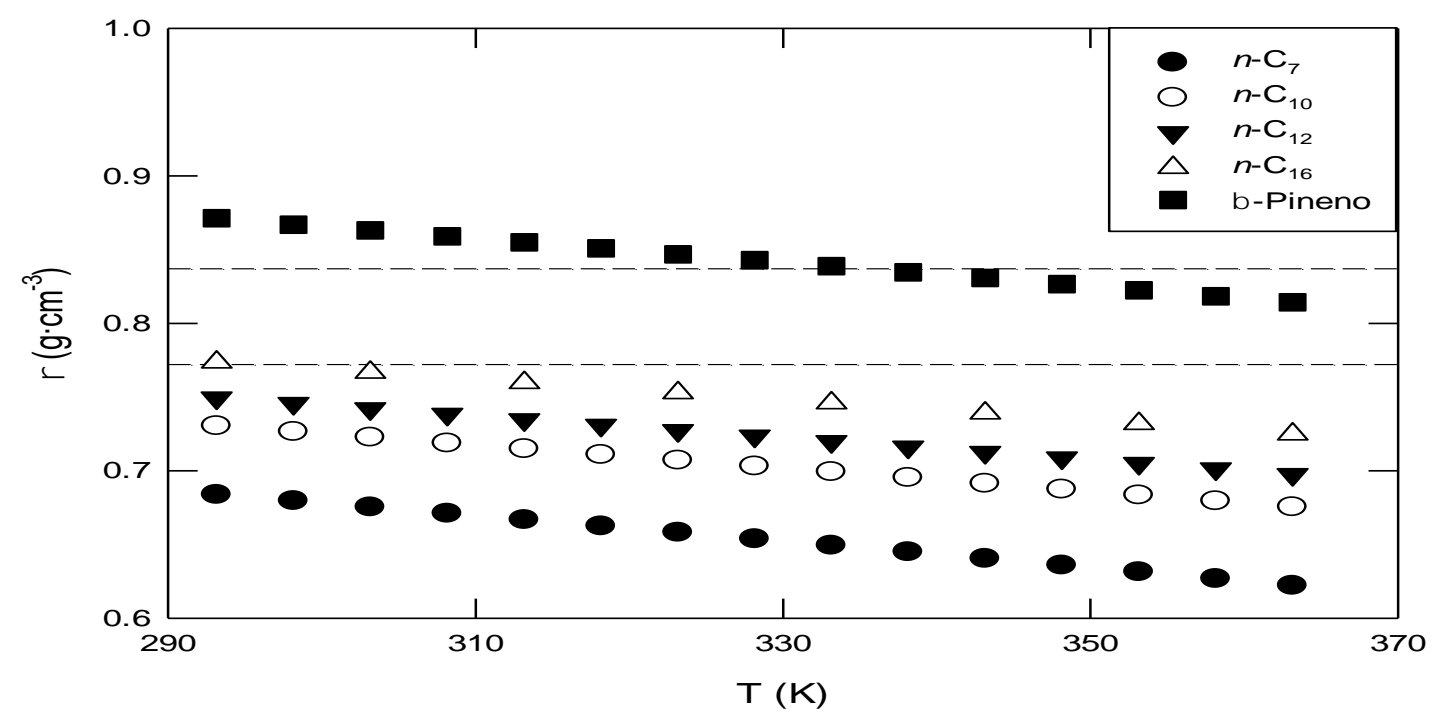

Figura 3. Densidad para diversos hidrocarburos y el $\beta$-pineno a diferentes temperaturas y presión atmosférica. Las líneas discontinuas representan los valores límites para la densidad de la turbosina a 293.15 K, de acuerdo con la NOM-016-CRE-2016. 
La viscosidad cinemática es una propiedad importante para el diésel (medida a $313.15 \mathrm{~K}$ ) y la turbosina (medida a $253.15 \mathrm{~K}$ ). En la Figura 4 se observa la viscosidad cinemática para los cuatro alcanos lineales y el $\beta$-pineno, la línea discontinua corresponde con los valores mínimo y máximo para esta propiedad en el diésel. Se observa que la única sustancia que cumple con este requisito, de acuerdo con la norma, es el $n$ - $\mathrm{C}_{16}$; el $\beta$-pineno se ubica por debajo del límite inferior establecido por la norma, es decir que el monoterpeno podría usarse como un aditivo, adicionándolo al diésel y así poder cumplir con la norma. Debido a las limitaciones técnicas, no fue posible medir la viscosidad a $253.15 \mathrm{~K}$.

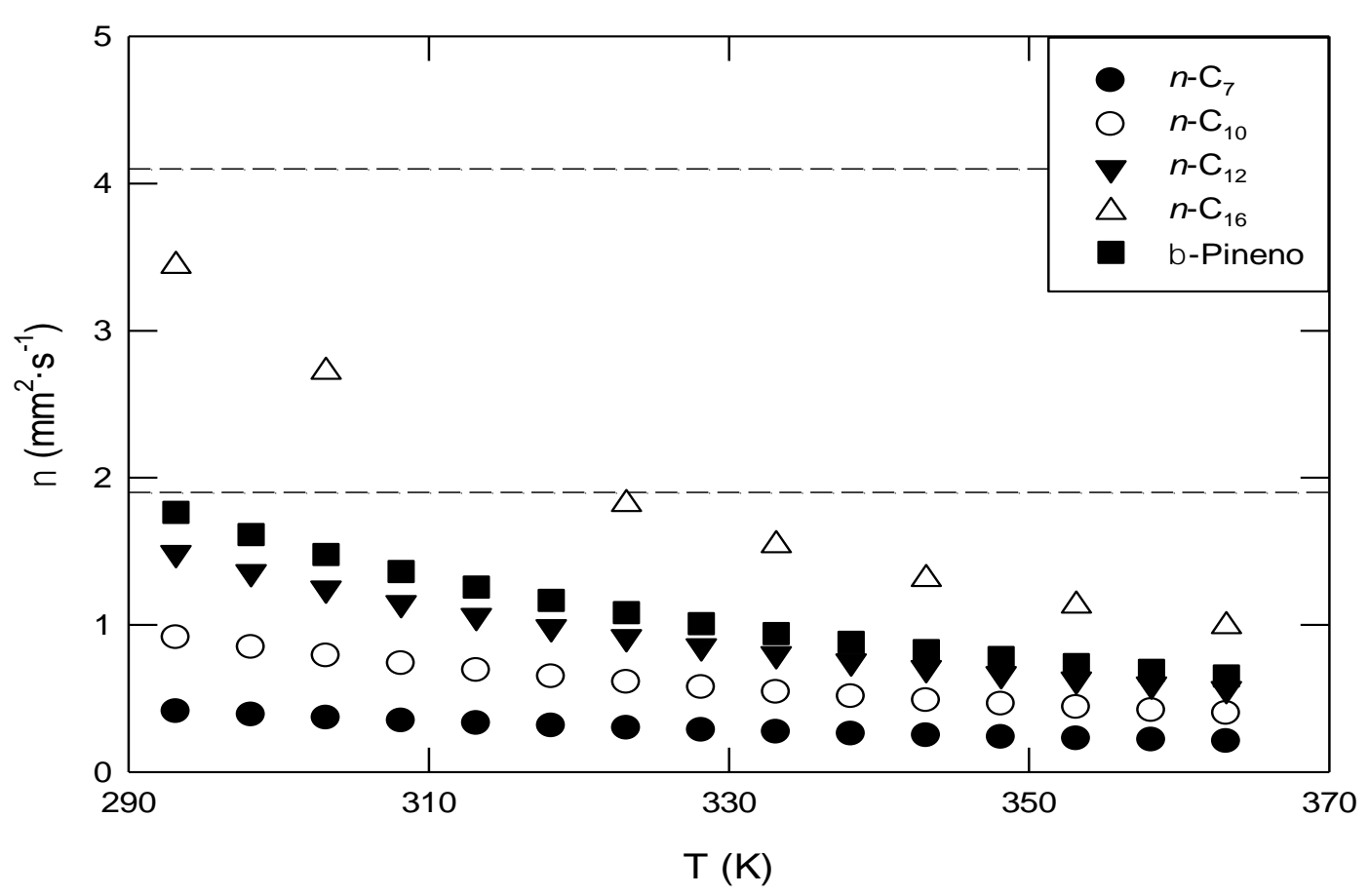

Figura 4. Viscosidad cinemática para hidrocarburos modelo de las gasolinas, diésel, turbosina y el $\beta$-pineno a diversas temperaturas y presión atmosférica. Las líneas discontinuas son los valores límites para la viscosidad cinemática del diésel a $313.15 \mathrm{~K}$, de acuerdo con la NOM-016-CRE-2016.

Finalmente, en la Figura 5 se muestran los valores del poder calorífico para los alcanos lineales y el $\beta$-pineno; la línea discontinua indica el valor mínimo del poder calorífico para petrolíferos de uso industrial y la línea continua es el mínimo para la turbosina. Se puede apreciar que todas las sustancias pueden ser utilizadas como combustibles de uso industrial y todas las parafinas cumplen con el valor mínimo para la turbosina. Los 
resultados apuntan a que el $\beta$-pineno pudiera ser utilizado como aditivo en los combustibles, en mezclas con los derivados de origen fósil.

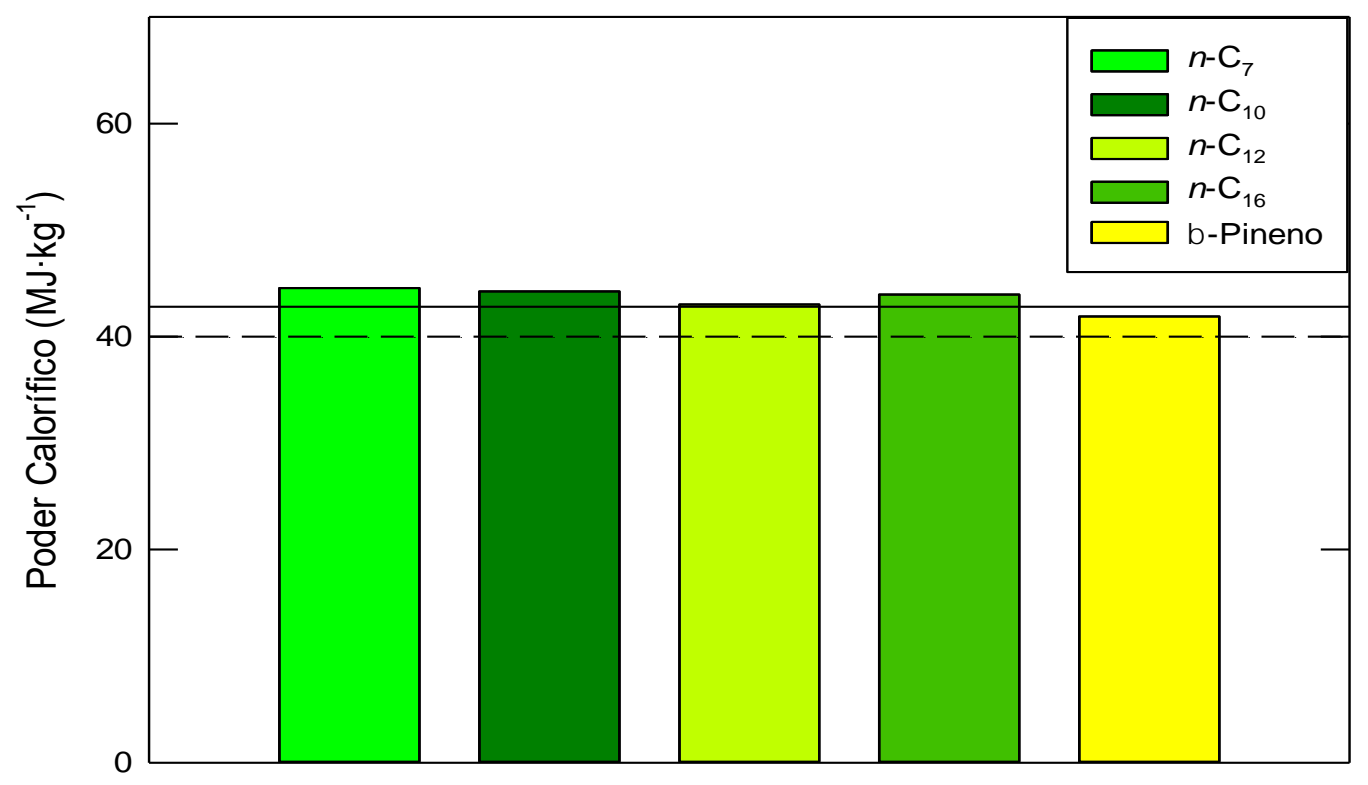

Figura 5. Poder calorífico para los hidrocarburos modelo de gasolina, diésel, turbosina y el $\beta$-pineno. La línea continua establece el valor mínimo para turbosinas y la línea discontinua establece el valor mínimo para los petrolíferos de uso industrial, de acuerdo con la NOM-016-CRE-2016.

\section{CONCLUSIÓN}

En este trabajo se llevó a cabo la comparación las propiedades fisicoquímicas de moléculas modelo para petrolíferos y el $\beta$-pineno; los resultados muestran que existen semejanzas en los valores obtenidos para la densidad, viscosidad cinemática y poder calorífico. Teniendo en cuenta lo anterior, el $\beta$-pineno tiene potencial para ser utilizado como aditivo en los combustibles derivados del petróleo. La principal ventaja del $\beta$ pineno es su proceso de producción, ya que no es necesario talar los pinos para obtenerlo, lo anterior puede ser un incentivo para los propietarios de tierras en zonas boscosas, al obtener un producto de valor agregado de los pinos usados en procesos de reforestación. Es necesaria la profundización de los estudios respecto al uso de los derivados de la trementina como biocombustibles, principalmente llevando a cabo pruebas en motores experimentales. 


\section{AGRADECIMIENTOS}

Los autores agradecen al Programa para el Desarrollo Profesional Docente (PRODEP) por el financiamiento recibido para la realización de este trabajo, a través del proyecto UJAT-PTC-291 y a la Universidad Juárez Autónoma de Tabasco por las facilidades otorgadas.

\section{LISTA DE REFERENCIAS}

Anand, B. P., Saravanan, C. G., \& Srinivasan, C. A. (2010). Performance and exhaust emission of turpentine oil powered direct injection diesel engine. Renewable Energy, 35(6), 1179-1184. https://doi.org/10.1016/j.renene.2009.09.010

Burgess, D. R. J. (2021). Thermochemical Data. In P. J. Linstrom \& W. G. Mallard (Eds.), NIST Chemistry WebBook, NIST Standard Reference Database No. 69. Gaithersburg MD, 20899: National Institute of Standards and Technology. Retrieved from https://doi.org/10.18434/T4D303

Comisión Reguladora de Energía. NOM-016-CRE-2016, Especificaciones de calidad de los petrolíferos. , (2016). México: Diario Oficial de la Federación.

Donoso, D., García, D., Ballesteros, R., Lapuerta, M., \& Canoira, L. (2021). Hydrogenated or oxyfunctionalized turpentine: Options for automotive fuel components. $\quad$ RSC Advances, 11(30), 18342-18350. https://doi.org/10.1039/d1ra03003e

Girón, S. (BBC T. (2021). Qué es el "oro líquido" que se esconde en los bosques de España. Retrieved December 21, 2021, from https://www.bbc.com/mundo/verttra-58936633

Honnet, S., Seshadri, K., Niemann, U., \& Peters, N. (2009). A surrogate fuel for kerosene. Proceedings of the Combustion Institute, 32 I(1), 485-492. https://doi.org/10.1016/j.proci.2008.06.218

Hudaya, T., Widjaja, O., Rionardi, A., \& Soerawidjaja, T. H. (2016). Synthesis of biokerosene through electrochemical hydrogenation of terpene hydrocarbons from turpentine oil. Journal of Engineering and Technological Sciences, 48(6), 655-664. https://doi.org/10.5614/j.eng.technol.sci.2016.48.6.2

Jung, J. K., Lee, Y., Choi, J. W., Jae, J., Ha, J. M., Suh, D. J., .. Lee, K. Y. (2016). Production of high-energy-density fuels by catalytic $\beta$-pinene dimerization: Effects of the catalyst surface acidity and pore width on selective dimer 
production. Energy Conversion and Management, 116, 72-79. https://doi.org/10.1016/j.enconman.2016.02.053

Knuuttila, P. (2013). Wood sulphate turpentine as a gasoline bio-component. Fuel, 104(2013), 101-108. https://doi.org/10.1016/j.fuel.2012.06.036

Lemmon, E. W., McLinden, M. O., \& Friend, D. G. (2021). Thermophysical Properties of Fluid Systems. In P. J. Linstrom \& W. G. Mallard (Eds.), NIST Chemistry WebBook, NIST Standard Reference Database No. 69. Gaithersburg MD, 20899: National Institute of Standards and Technology. https://doi.org/https://doi.org/10.18434/T4D303

Luning Prak, D. J., Cowart, J. S., McDaniel, A. M., \& Trulove, P. C. (2016). Density, Viscosity, Speed of Sound, Bulk Modulus, Surface Tension, and Flash Point of Binary Mixtures of Butylcyclohexane with Toluene or n-Hexadecane. Journal of Chemical and Engineering Data, 61(10), 3595-3606. https://doi.org/10.1021/acs.jced.6b00516

Martins, J., \& Brito, F. P. (2020). Alternative fuels for internal combustion engines. Energies, 13(15). https://doi.org/10.3390/en13164086

Mata, N. T., Villanueva, S., \& Henríquez, M. (2018). Trend study: Applications of Colophony ( Rosin ) and its Derivatives. Ingeniería UC, 25 (3), 325-337. Retrieved from https://www.redalyc.org/jatsRepo/707/70757670002/70757670002.pdf

Mirov, N. T., Zavarin, E., \& Snajberk, K. (1966). Chemical composition of the turpentines of some Eastern Mediterranean pines in relation to their classification. Phytochemistry, 5(1), 97-102. https://doi.org/10.1016/S0031-9422(00)85086-4

National Center for Biotechnology Information. (2021a). PubChem Compound Summary for CID 10290825, (+)-beta-Pinene. Retrieved December 21, 2021, from https://pubchem.ncbi.nlm.nih.gov/compound/10290825

National Center for Biotechnology Information. (2021b). PubChem Compound Summary for CID 440967, (-)-beta-Pinene. Retrieved December 21, 2021, from https://pubchem.ncbi.nlm.nih.gov/compound/440967

National Center for Biotechnology Information. (2021c). PubChem Compound Summary for CID 440968, (-)-alpha-Pinene. Retrieved December 21, 2021, from https://pubchem.ncbi.nlm.nih.gov/compound/1S_-_-_-alpha-Pinene 
National Center for Biotechnology Information. (2021d). PubChem Compound Summary for CID 82227, (+)-alpha-Pinene. Retrieved December 21, 2021, from https://pubchem.ncbi.nlm.nih.gov/compound/1R_-_-alpha-Pinene

Pitz, W. J., Cernansky, N. P., Dryer, F. L., Egolfopoulos, F. N., Farrell, J. T., Friend, D. G., \& Pitsch, H. (2007). Development of an experimental database and chemical kinetic models for surrogate gasoline fuels. SAE Technical Papers, 1-21. https://doi.org/10.4271/2007-01-0175

Toledo, A., \& Chávez, A. (2006). Resina: entre la madera y el desarrollo comunitario integral. Biodiversitas, (65), 1-7. 